\title{
Non-enzymatic glucose biosensor based on hyperbranched pine-like gold
}

\section{nanostructure}

\author{
H. Heli ${ }^{*}$, O. Amirizadeh
}

Nanomedicine and Nanobiology Research Center, Shiraz University of Medical Sciences, Shiraz, Iran.

Department of Nanomedicine, School of Advanced Medical Sciences and Technologies, Shiraz University of Medical Sciences, Shiraz, Iran.

*Corresponding author. E-mail: hheli7@yahoo.com, heli@sums.ac.ir (H. Heli), Tel, Fax: +98 7136281506 .

\begin{abstract}
Hyperbranched pine-like gold nanostructure was electrodeposited on the polycrystalline gold surface at $0 \mathrm{mV}$ (vs. $\mathrm{AgCl}$ ) with the assistance of histidine as a soft template. The nanostructure was then applied as a highly sensitive nonenzymatic sensor for glucose. The catalytic activity and sensitivity of the gold nanostructure toward the electrooxidation of glucose was excellent without surface fouling and deterioration effects. The current related to the oxidation of glucose rapidly and linearly depended on its concentration with a sensitivity of $776.8 \mu \mathrm{A} \mathrm{cm} \mathrm{mmol}^{-1}$ $\mathrm{dm}^{3}$, a detection limit of $3.39 \mu \mathrm{mol} \mathrm{dm}{ }^{-3}$ with a relative standard deviation of $2.32 \%$.
\end{abstract}

Keywords: Nonenzymatic biosensor; Glucose; Gold; Hierarchical nanostructure

\section{Introduction}

Development of simple, reliable and fast methods for detection of glucose is important in many fields such as clinical diagnostics, and biotechnological and food 
industries. In addition, determination of glucose in the blood is of great importance for diabetes screening and treatment. On the other hand, study of the electrooxidation of glucose on different electrode materials is an attractive research area for applications in glucose fuel cells, and for development of electrochemical glucose sensors and biosensors $[1,2]$.

Enzymatic glucose biosensors suffer from some disadvantages such complicated immobilization procedure, long-term instability, and high cost. Therefore, considerable research has focused on development of non-enzymatic glucose sensors based on direct electrooxidation of glucose on the electrode surface without using any enzyme. Non-enzymatic glucose sensors present the advantages of stability, reproducibility, low cost, and freedom from oxygen limitation [3]. However, these glucose sensors suffer from slow kinetics, lack of selectivity, and fouling of the surface. To overcome these drawbacks, nanomaterials have been applied as a sudden surge in the fabrication of non-enzymatic glucose sensors. Nanomaterials possess a high real surface area, accelerate the kinetics (of both mass transport and charge transfer processes) of the glucose electrooxidation, and therefore, provide high sensitivity for glucose detection. There have been developed glucose sensors using different nanomaterials including platinum, gold, palladium, and metal oxides $[1,4-$ 6]. Among them, due to biocompatibility, higher activity, and lower potential of glucose electrooxidation, gold is one of the most inspiring materials for the fabrication of glucose sensors [7-26].

In the present study, a non-enzymatic electrochemical glucose biosensor based on hyperbranched pine-like gold nanostructure was presented for a rapid, simple and quantitative detection of glucose. 


\section{Experimental section}

All chemicals used in this work were reagent grade from Merck (Darmstadt, Germany) or Sigma (St. Loius, MO, USA) products and used without further purifications. Doubly distilled water was used throughout.

Electrochemical measurements were performed in a conventional three-electrode cell powered by a $\mu$-Autolab potentiostat/galvanostat, type III, FRA2 (The Netherlands) run by a computer through GPES 4.9 commercial software. An $\mathrm{Ag} / \mathrm{AgCl} / 3 \mathrm{~mol} \mathrm{dm}{ }^{-3} \mathrm{KCl}$ and a platinum plate were used as the reference and counter electrodes, respectively. The working electrode was a gold disk ( $\mathrm{Au}$ ) electrode of 2 mm diameter covered by hyperbranched pine-like gold nanostructure (HP-Au). Studies on the electrooxidation and determination of glucose were performed in 100 mmol $\mathrm{dm}^{-3}$ sodium hydroxide solution as the running electrolyte.

In order to prepare HP-Au electrode, the Au electrode was firstly polished by sand papers and then lubricated by glycerin on a polishing pad with $50 \mathrm{~nm}$-alumina powder. Polishing was continued to attain a mirror-like surface. The electrode was then cleaned by immersion in a 1:3 water/ethanol mixture and ultrasonication for 5 min in an ultrasound bath. The electrode was further electropolished by immersion in a $500 \mathrm{mmol} \mathrm{L}^{-1} \mathrm{H}_{2} \mathrm{SO}_{4}$ solution, applying cyclic potentials in the range of cathodic and anodic edges of the electrolyte for 25 consecutive cycles. The Au electrode was then placed in the cell containing $20 \mathrm{mmol} \mathrm{L}^{-1} \mathrm{HAuCl}_{4}+0.5 \mathrm{~mol} \mathrm{~L}^{-1} \mathrm{H}_{2} \mathrm{SO}_{4}+150$ mmol $\mathrm{L}^{-1}$ histidine. Hyperbranched pine-like gold nanostructure was electrodeposited at a potential of $0.0 \mathrm{mV}$ in a duration of $600 \mathrm{~s}$. HP-Au electrode was then rinsed thoroughly with distilled water.

The real surface area of the HP-Au electrode was measured electrochemically. The HP-Au electrode was transferred to a solution of $\mathrm{KCl}\left(0.5 \mathrm{~mol} \mathrm{dm}^{-3}\right)$ containing 
$\mathrm{K}_{4}\left[\mathrm{Fe}(\mathrm{CN})_{6}\right]\left(0.5 \mathrm{mmol} \mathrm{dm}^{-3}\right)$, and cyclic voltammograms were measured at different potential sweep rates. Using the Randles-Sevcik equation [27] and the value of $7.60 \times 10^{-6} \mathrm{~cm} \mathrm{~s}^{-1}$ for the diffusion coefficient of $\left[\mathrm{Fe}(\mathrm{CN})_{6}\right]^{4-}[28]$, the real surface area of the HP-Au electrode was obtained.

In order to obtain information about the morphology and size of the electrodeposited gold nanostructure, field emission scanning electron microscopy (FESEM) was performed by the instrument Zeiss, Sigma-IGMA/VP (Germany).

Amperometric measurements for the glucose quantitation were performed at constant potential of $70 \mathrm{mV}$ in a magnet-stirring solution wherein the solution was rapidly homogenized after each glucose injection.

In order to investigate the capability of the sensor to detect glucose in real samples, four blood serum samples taken from different persons were analyzed by the amperometry method. All studies were carried out at room temperature.

\section{Results and discussion}

Fig. 1 presents FESEM images of the HP-Au electrode surface with different magnifications. The images show a dendritic morphology with different sizes of hyperbranched pine-like. The dendrites themselves comprise nanoparticles of 150-200 nm. Therefore, the dendritic nanostructure electrodeposited in the presence of histidine has a hierarchical structure. The real surface area of the HPAu electrode (supplementary material $\mathrm{S} 1$ ) was obtained as $0.26 \mathrm{~cm}^{-2}$, while the geometric surface area was $0.0314 \mathrm{~cm}^{-2}$ indicating a roughness factor of 8.2. The surface texture of the HP-Au electrode provides a large overall surface area for the electrooxidation of glucose. 
Fig. 2 shows cyclic voltammograms of $\mathrm{Au}$ and HP-Au electrodes in the absence and presence of glucose. Glucose is electrooxidized on the HP-Au electrode surface at (at least $120 \mathrm{mV}$ ) lower potentials than the Au electrode. In addition, the rate of the glucose electrooxidation on the HP-Au electrode is about 6.3 times higher than the $\mathrm{Au}$ electrode. Regarding the roughness factor of the HP-Au electrode, and its comparison with the enhancement in the rate of electrooxidation process, it can be deduced that the increment in the current can be related to the increment in the real surface area of the HP-Au electrode. However, comparison of the potentials of the electrooxidation process on the electrode surfaces indicates that HP-Au electrode can favor the electrooxidation to occur at lower energies. Therefore, HP-Au electrode accelerates the electrooxidation of glucose from thermodynamic point of view arising from the nano-size and/or nano-shape effects. Both aspects are highly important for the development of a determination method of glucose with high sensitivity without interference from other compounds.

Fig. 3 shows the typical pseudo-steady state polarization curve for the electrooxidation of glucose on the HP-Au electrode surface representing a S-shape curve. The corresponding Tafel plot is shown in the inset. Using the Tafel plot, the electron transfer coefficient of the electrooxidation of glucose was obtained as 0.41 .

In order to develop a method for glucose determination using HP-Au electrode, amperometric signals were recorded. Figure 4 shows the typical amperometric signals during the successive addition of glucose to the running electrolyte at the potential of $70 \mathrm{mV}$. Gentle magnetic stirring for a few seconds was needed to homogenize the solution after each addition. The HP-Au electrode responded rapidly, and the linear dependency of the transient current on the glucose concentration is shown in Fig. 4, inset. The limits of detection (LOD) and quantitation (LOQ) of the method were 
calculated as $3 \mathrm{SD} / \mathrm{m}$ and $10 \mathrm{SD} / \mathrm{m}$, respectively, where $\mathrm{SD}$ is the standard deviation of the blank signal and $\mathrm{m}$ is the slope of the calibration curve [29]. The determined parameters for the calibration curve of glucose were obtained as LOD $=3.39 \mu \mathrm{mol} \mathrm{dm}-$ ${ }^{3}$, LOQ $=11.30 \mu \mathrm{mol} \mathrm{dm}{ }^{-3}$, R.S.D. $=2.32 \%$, sensitivity $=776.8 \mu \mathrm{A} \mathrm{cm}^{-2} \mathrm{mmol}^{-1} \mathrm{dm}^{3}$, and a linear range of $20-240 \mu \mathrm{mol} \mathrm{dm}{ }^{-3}$. A comparison of some different non-enzymatic glucose sensors based on different gold structures is presented in Table 1 and it shows that the present sensor has better or comparable characteristics.

In order to inspect the selectivity of the HP-Au electrode, the interference effects of theophylline, lysine, methionine, histidine, xanthine, arginine and ascorbic acid were evaluated and shown in Fig.5. Under the experimental conditions applied in this work, no chemical interference was observed for all these compounds. This is due to the applied potential for the electrooxidation of glucose; the potential was too negative for these compounds to oxidize.

In order to investigate the capability of the sensor to detect glucose in real samples, four blood serum samples were analyzed by amperometry. The values of glucose levels in serum samples obtained by the HP-Au electrode are reported in Table 2.

In order to verify the durability and stability of the Pt/ HP-Au electrode, similar amperometric measurements were performed in five days. It was found that the currents changed slightly $(<4.1 \%)$. During the analysis, no gold particle was observed in the analyzed solutions and no visual change was observed on the electrode surface. Because the gold particles were hierarchically electrodeposited, without mechanical abrasion, the HP-Au electrode remained stable. In addition, the electrode was rinsed by distilled water and stored in distilled water when not in use. 


\section{Conclusion}

A green electrodeposition was developed for the synthesis of hyperbranched pine-like gold nanostructure on gold surfaces by controlling the deposition potential in the presence of histidine. The synthesis procedure can be expanded to the synthesis of other noble metal nanostructures. The hyperbranched pine-like gold nanostructure represented an enhanced electrocatalytic activity toward the oxidation of glucose at potentials much more negative than some other gold-based electrodes. Voltammetric and amperometric measurements showed that the nanostructure can be used as a nonenzymatic glucose sensor with a low detection limit, high selectivity and sensitivity.

\section{Acknowledgments}

We would like to thank the Research Council of Shiraz University of Medical Sciences (8885), and also the Iran National Science Foundation (INSF) for supporting this research.

\section{References:}

[1] A.A. Saei, P. Najafi-Marandi, A. Abhari, M. de la Guardia, J.E.N. Dolatabadi, Electrochemical biosensors for glucose based on metal nanoparticles, Trends Anal. Chem. 42 (2013) 216-227.

[2] J. Wang, Electrochemical glucose biosensors, Chem. Rev. 108 (2008) 814-825.

[3] S. Park, H. Boo, T.D. Chung, Electrochemical non-enzymatic glucose sensors, Anal. Chim. Acta 556 (2006) 46-57.

[4] N. Sattarahmady, H. Heli, A non-enzymatic amperometric sensor for glucose based on cobalt oxide nanoparticles, J. Exp. Nanosci. 7 (2012) 529-546. 
[5] H.H. Ai, X.T. Huang, Z.H. Zhu, J.P. Liu, Q.B. Chi, Y.Y. Li, Z.K. Li, X.X. Ji, A novel glucose sensor based on monodispersed $\mathrm{Ni} / \mathrm{Al}$ layered double hydroxide and chitosan, Biosens. Bioelectron. 24 (2008) 1048-1052.

[6] L-C. Jiang, W-D. Zhang, A highly sensitive nonenzymatic glucose sensor based on $\mathrm{CuO}$ nanoparticles-modified carbon nanotube electrode, Biosens. Bioelectron. 25 (2010) 1402-1407.

[7] H. Shu, G. Chanf, Z. Wang, P. Li, Y. Zhang, Y. He, Pulse laser deposition fabricating gold nanoclusters on a glassy carbon surface for nonenzymatic glucose sensing, Anal. Sci. 31 (2015) 609-616.

[8] G. Chang, H. Shu, K. Ji, M. Oyama, X. Liu, Y. He, Gold nanoparticles directly modified glassy carbon electrode for non-enzymatic detection of glucose, Appl. Surf. Sci. 288 (2014) 524-529.

[9] E.H. El-Ads, A. Galal, N.F. Atta, Electrochemistry of glucose at gold nanoparticles modified graphite/ $\mathrm{SrPdO}_{3}$ electrode-Towards a novel non-enzymatic glucose sensor, J. Electroanal. Chem. 749 (2015) 42-52.

[10] Y. Zhao, J. Chu, S-H. Li, W-W. Li, G. Liu, Y-C. Tian, H-Q. Yu, Non-enzymatic electrochemical detection of glucose with a gold nanowire array electrode, Electroanalysis 26 (2014) 656-663.

[11] H. Shu, L. Cao, G. Chang, H. He, Y. Zhang, Y. He, Direct electrodeposition of gold nanostructures onto glassy carbonelectrodes for non-enzymatic detection of glucose, Electrochim. Acta 132 (2014) 524-532.

[12] N.S. Ismail, Q.H. Le, H. Yoshikawa, M. Saito, E. Tamiya, Development of nonenzymatic electrochemical glucose sensorbased on graphene oxide nanoribbongold nanoparticle hybrid, Electrochim. Acta 146 (2014) 98-105. 
[13] K-J. Chen, W-N. Su, C-J. Pan, S-Y. Cheng, J. Rick, S-H. Wang, C-C. Liu, C-C. Chang, Y-W. Yang, C-H. Wang, B-J. Hwang, Dendritic platinum-decorated gold nanoparticles for non-enzymatic glucose biosensing, J. Mater. Chem. B 1 (2013) $5925-5932$.

[14] Y-G. Zhou, S. Yang, Q-Y. Qian, X-H. Xia, Gold nanoparticles integrated in a nanotube array for electrochemical detection of glucose, Electrochem. Commun. 11 (2009) 216-219.

[15] T-M. Cheng, T-K. Huang, H-K. Lin, S-P. Tung, Y-L. Chen, C-Y. Lee, H-T. Chiu, (110)-Exposed gold nanocoral electrode as low onset potential selective glucose sensor, ACS Appl. Mater. Interfaces, 2 (2010) 2773-2780.

[16] M. Wooten, J. H. Shim, W. Gorski, Amperometric determination of glucose at conventional vs. nanostructured gold electrodes in neutral solutions, Electroanalysis 22 (2010) 1275-1277.

[17] Y. Li, Y-Y. Song, C. Yang, X-H. Xia, Hydrogen bubble dynamic template synthesis of porous gold for nonenzymatic electrochemical detection of glucose, Electrochem. Commun. 9 (2007) 981-988.

[18] R. Prehn, M. Cortina-Puig, F.X. Munoz, A non-enzymatic glucose sensor based on the use of gold micropillar array electrodes, J. Electrochem. Soc. 159 (2012) F134-F139.

[19] Y.G. Zhou, S. Yang, Q.Y. Qian, X.H. Xia, Gold nanoparticles integrated in ananotube array for electrochemical detection of glucose, Electrochem. Commun. 11 (2009) 216-219.

[20] H. Zhang, J. Xu, H. Chen, Shaped-controlled gold nanoarchitectures: synthesis, superhydrophobicity, and electrocatalytic properties, J. Phys. Chem. C 112 (2008) 13886-13892. 
[21] M. Gougis, A. Tabet-Aoul, D. Ma, M. Mohamedi, Nanostructured cerium oxide catalyst support: Effects of morphology on the electroactivity of gold toward oxidative sensing of glucose, Microchim. Acta 181 (2014) 1207-1214.

[22] A. Liu, E. Liu, G. Yang, N.W. Khun, W. Ma, Non-enzymatic glucose detection using nitrogen-doped diamond-like carbon electrodes modified with gold nanoclusters, Pure Appl. Chem. 82 (2010) 2217-2229.

[23] D. Feng, F. Wang, Z. Chen, Electrochemical glucose sensor based on one-step construction of gold nanoparticle-chitosan composite film, Sens. Actuat. B 138 (2009) 539-544.

[24] S. Cherevko, C-H. Chung, Gold nanowire array electrode for non-enzymatic voltammetric and amperometric glucose detection, Sens. Actuat. B 142 (2009) 216-223.

[25] A. Liu, Q. Ren, T. Xu, M. Yuan, W. Tang, Morphology-controllable gold nanostructures on phosphorus doped diamond-like carbon surfaces and their electrocatalysis for glucose oxidation, Sens. Actuat. B 162 (2012) 135-142.

[26] M. Gougis, A. Tabet-Aoul, D. Ma, M. Mohamedi, Laser synthesis and tailordesign of nanosized gold onto carbonnanotubes for non-enzymatic electrochemical glucose sensor, Sens. Actuat. B 193 (2014) 363-369.

[27] A.J. Bard, L.R. Faulkner, Electrochemical methods: fundamentals and applications. John Wiley \& Sons, Inc, 2001, 156-176.

[28] S.F. Wang, Q. Xu, Electrochemical parameters of ethamsylate at multi-walled carbon nanotube modified glassy carbon electrodes, Bioelectrochem. 70 (2007) 296-300.

[29] J.C. Miller, J.N. Miller, Statistics for Analytical Chemistry, fourth ed., EllisHarwood, New York, 1994. 


\section{Figure legends:}

Fig. 1: FESEM images of the HP-Au electrode surface.

Fig. 2: Cyclic voltammograms of $\mathrm{Au}$ and HP-Au electrodes in the absence and presence of $0.2 \mathrm{mmol} \mathrm{dm}{ }^{-3}$ glucose.

Fig. 3: Typical pseudo-steady state polarization curve for the electrooxidation of 0.2 mmol dm ${ }^{-3}$ glucose on the HP-Au electrode. Inset: The corresponding Tafel plot.

Figure 4: Typical amperometric signals during the successive addition of glucose to the running electrolyte at the potential of $70 \mathrm{mV}$. Inset: The calibration curve.

Fig. 5: Typical amperometric signals during the successive addition of glucose, theophylline, lysine, methionine, histidine, xanthine, arginine and ascorbic acid with the concentration of $20 \mu \mathrm{mol} \mathrm{dm}{ }^{-3}$. 

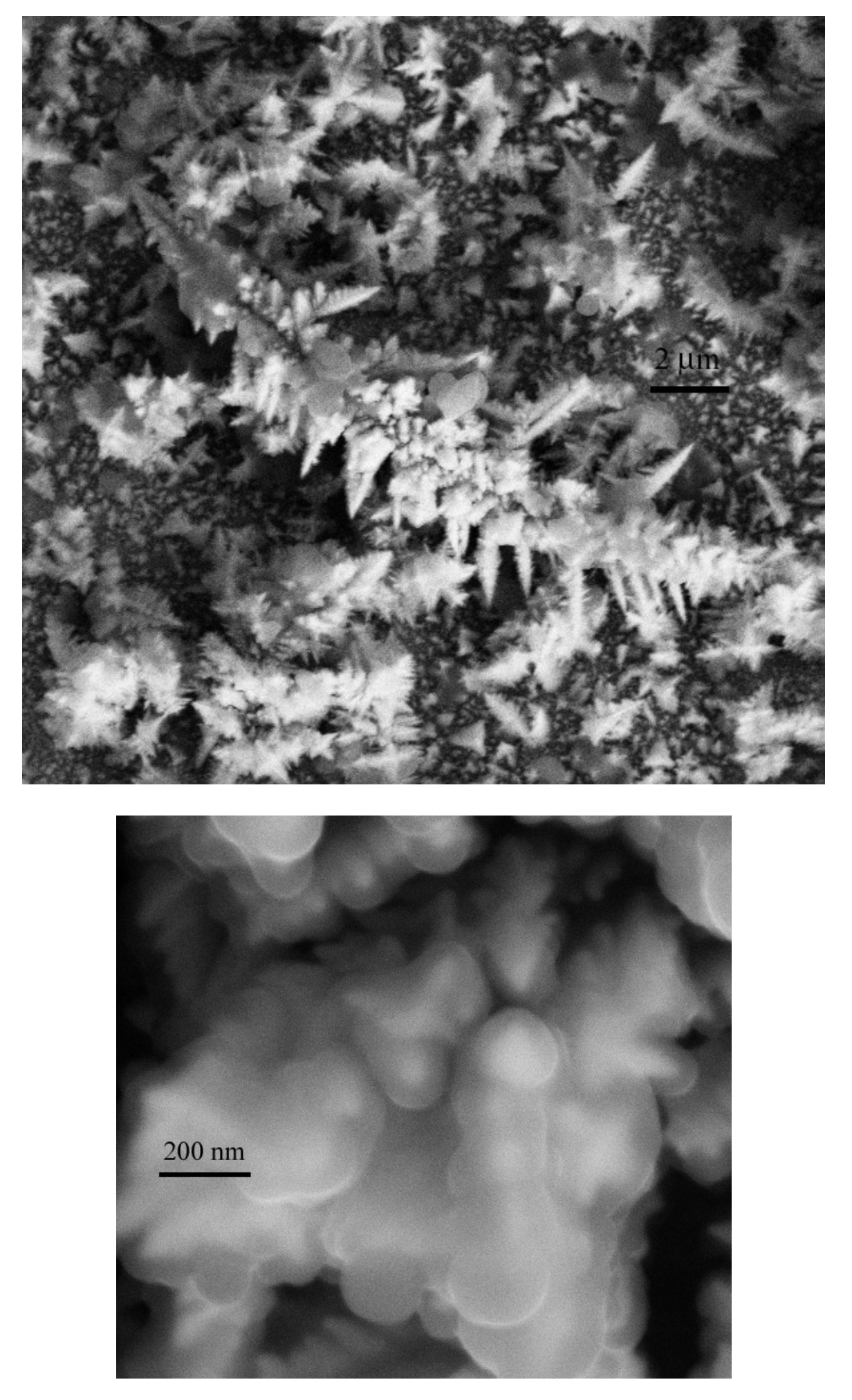

Figure 1

\section{Fire}

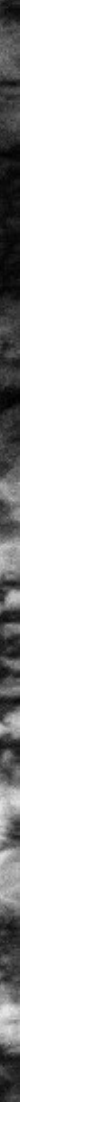
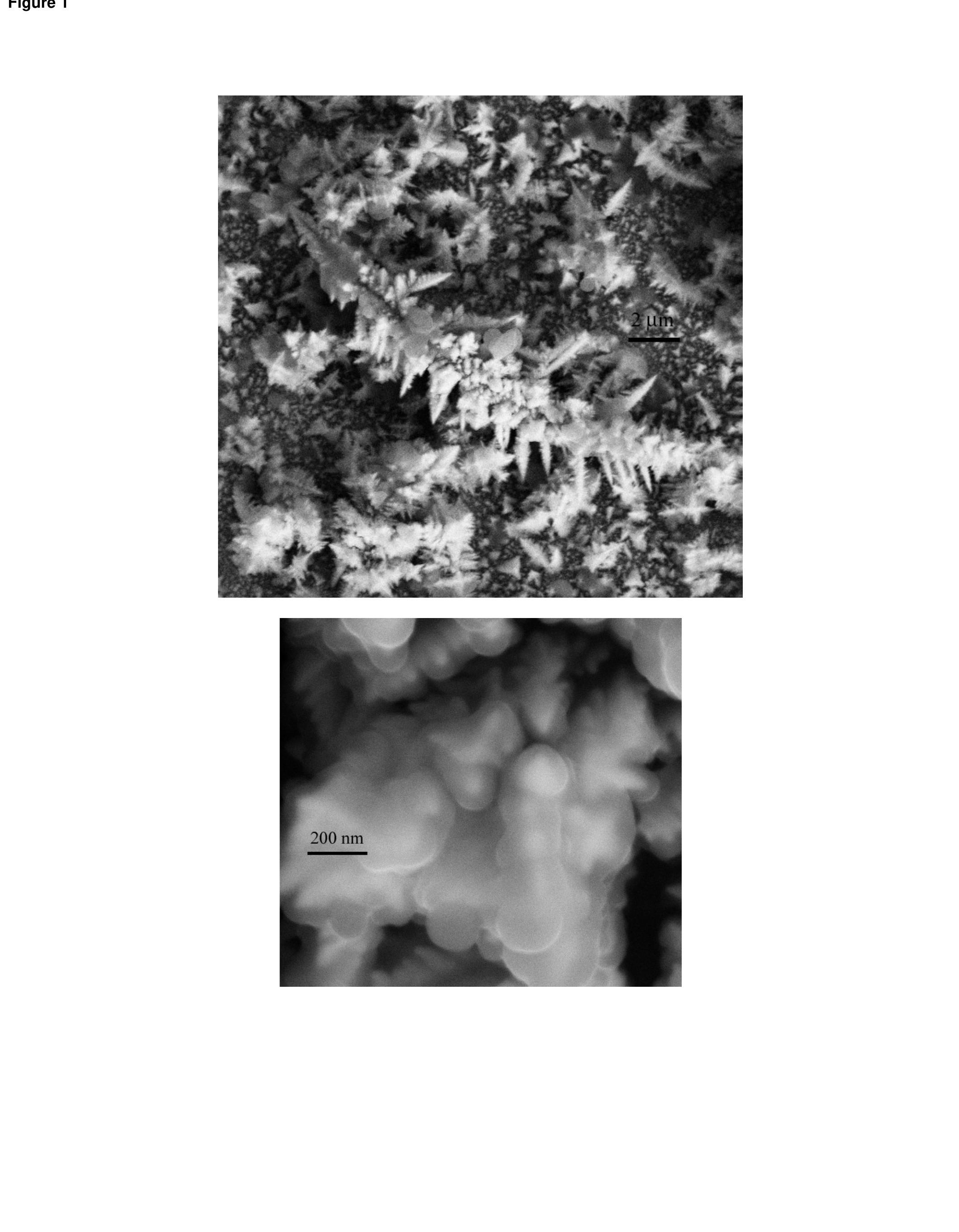

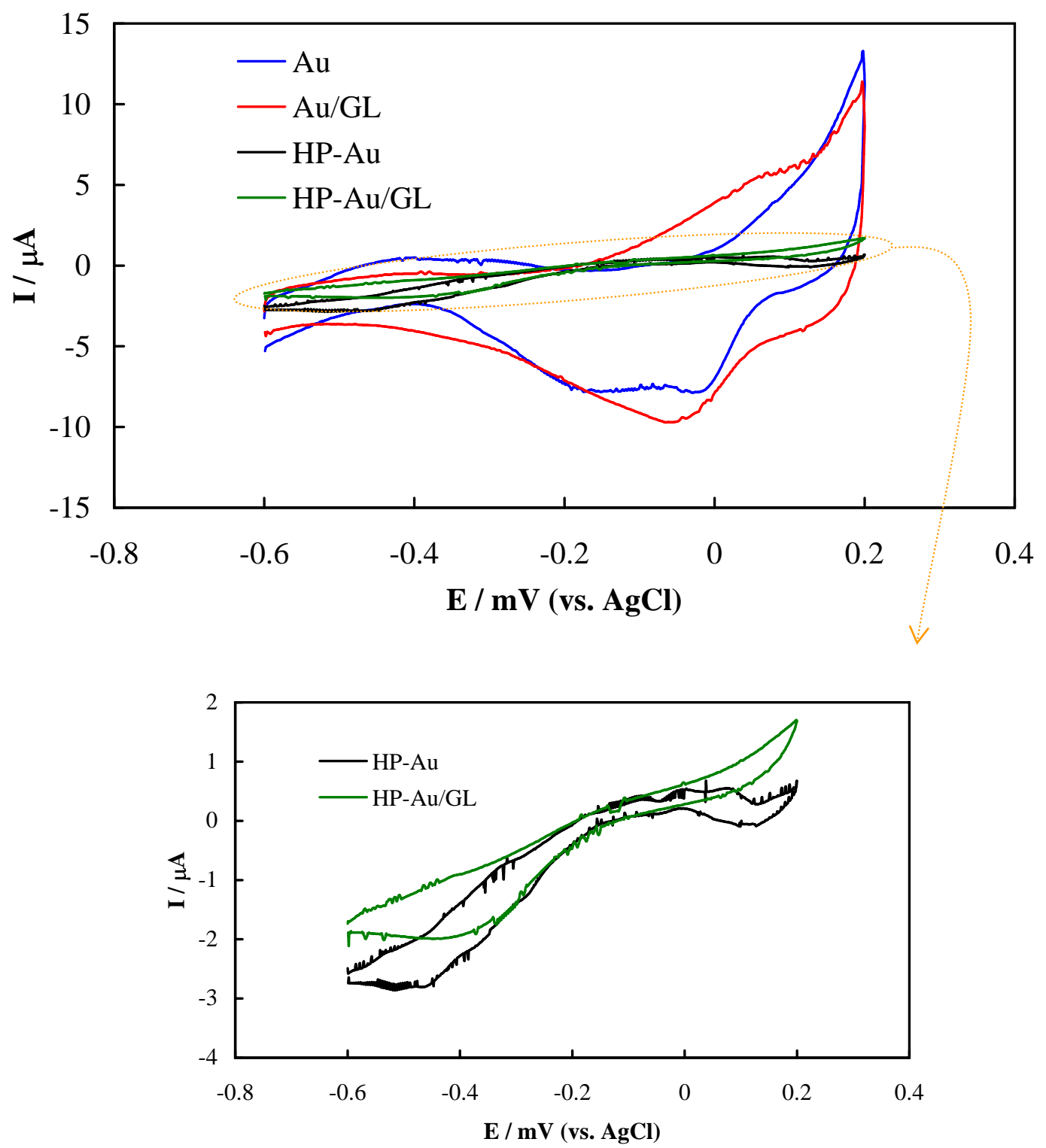


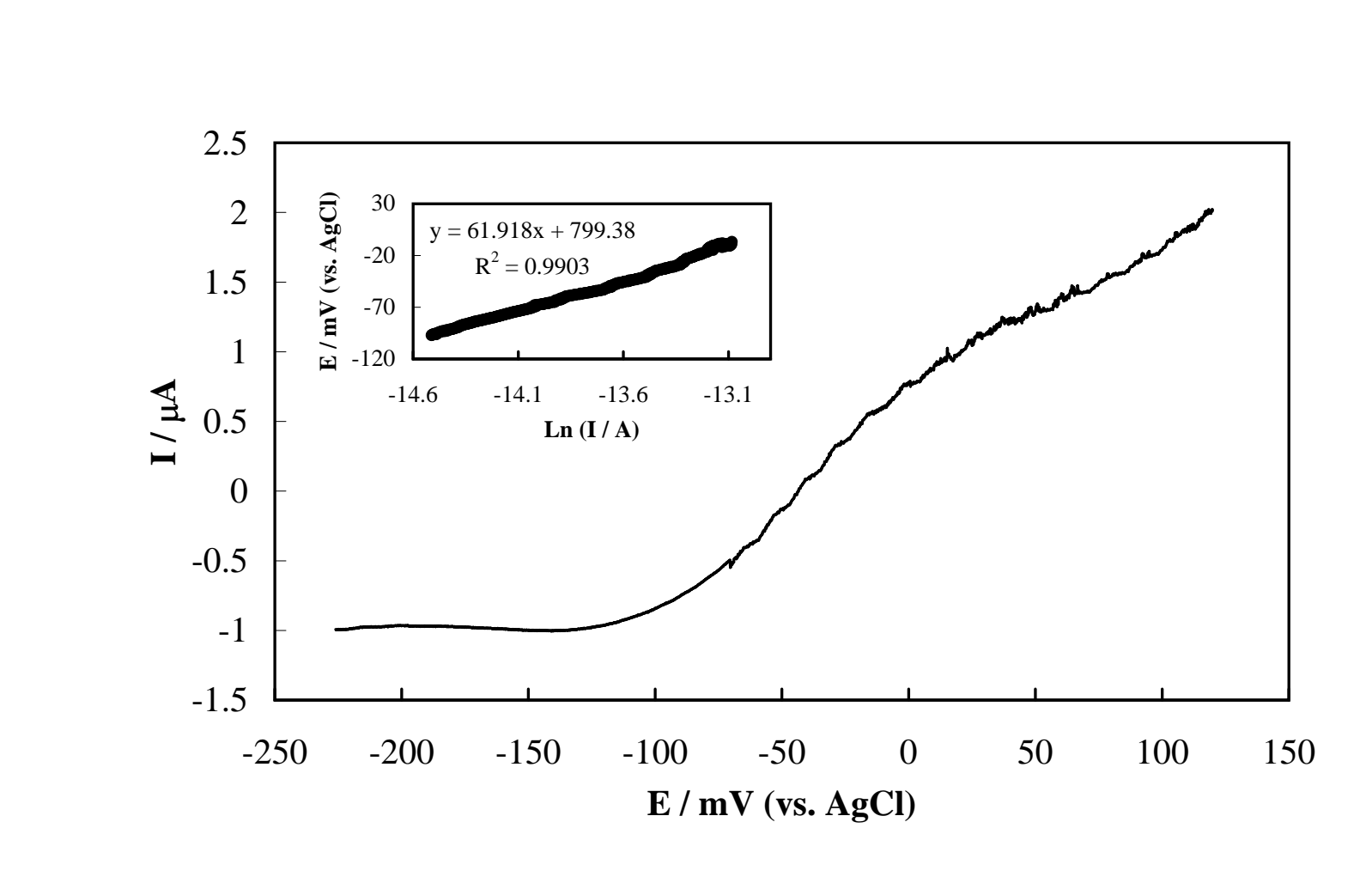

Figure 3

.

3

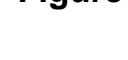
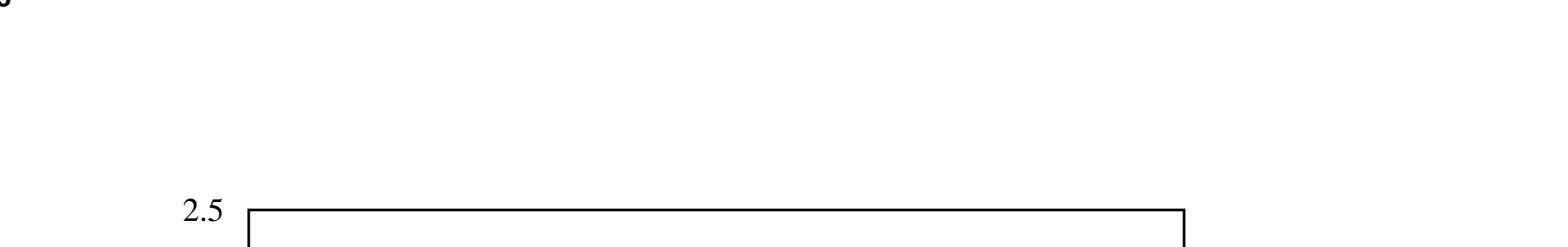

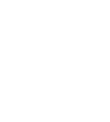

.
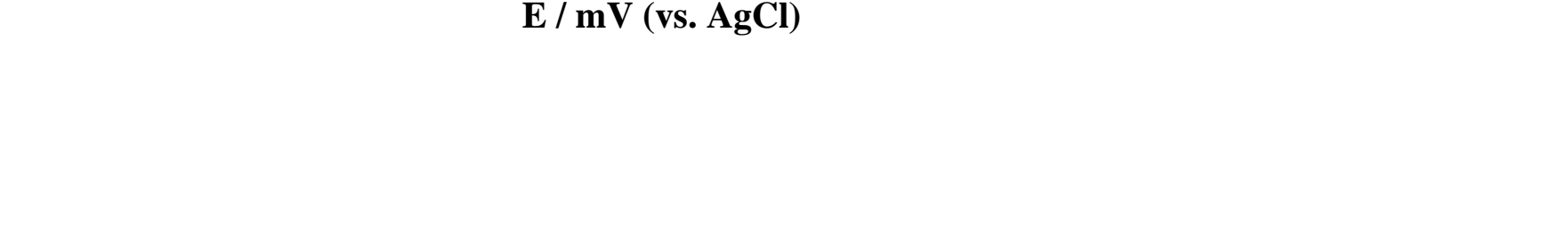


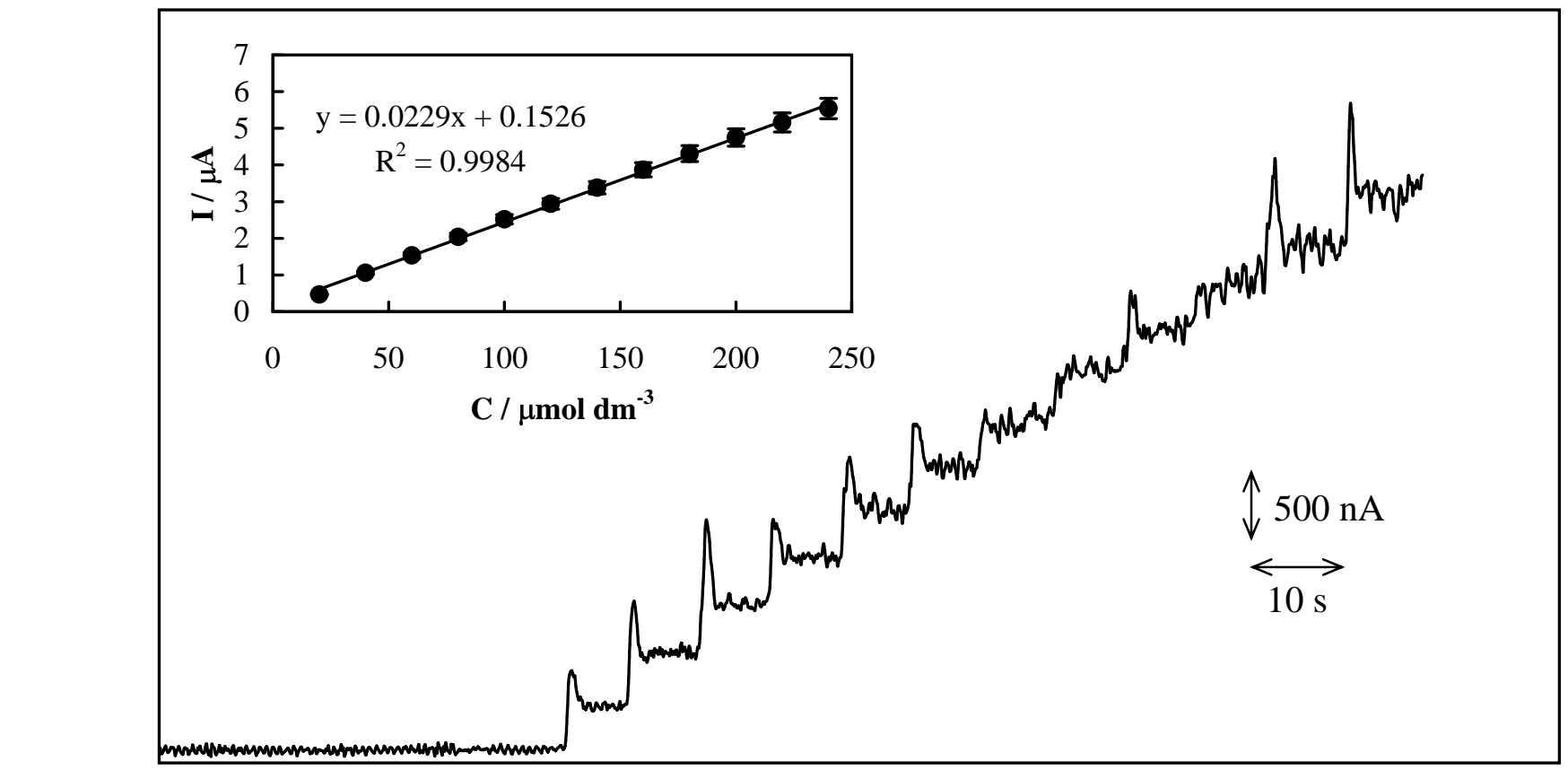



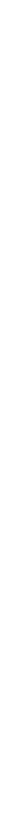

Uning

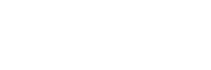




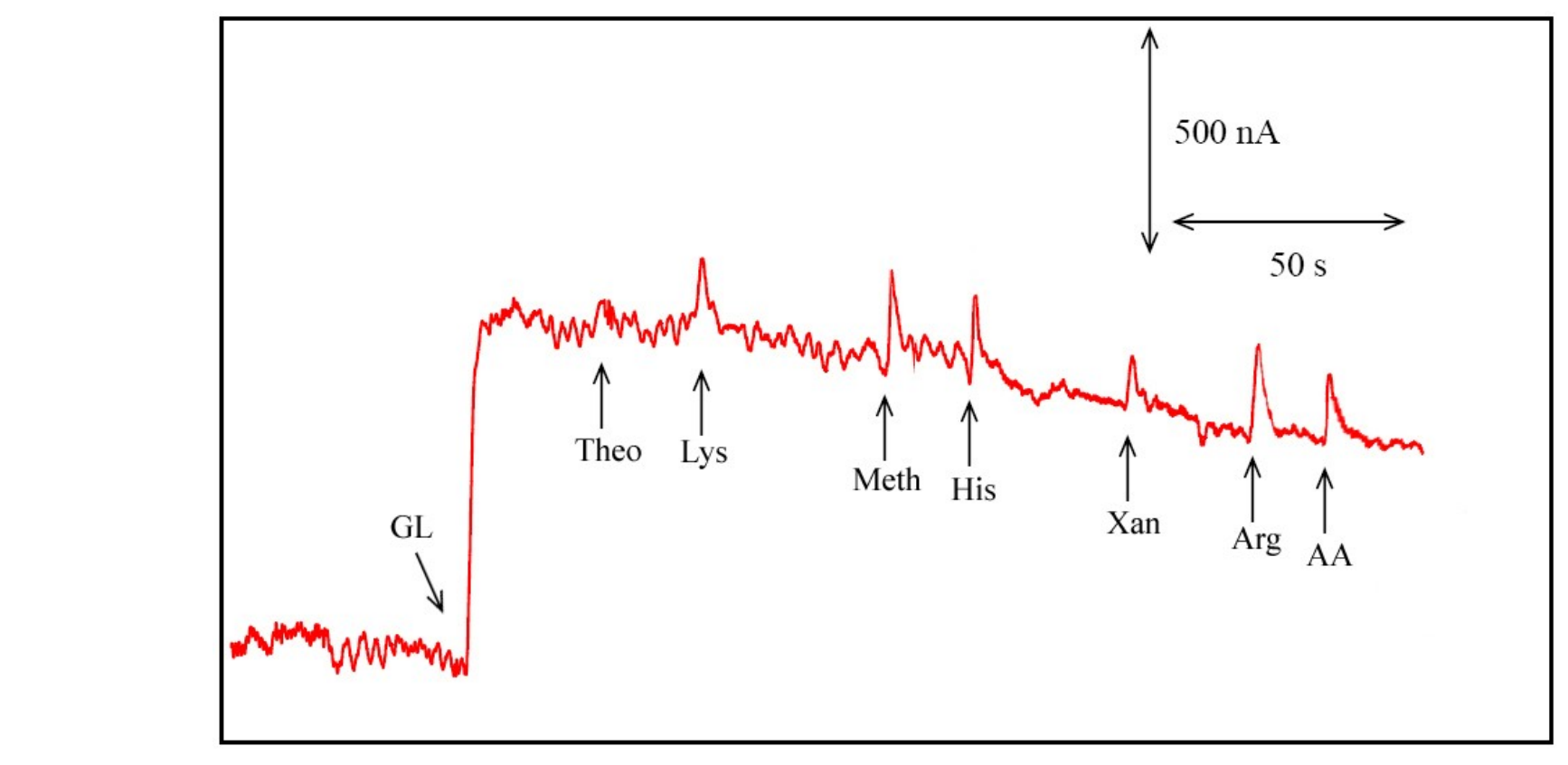

Figure 5

$$
\text { (1) }
$$

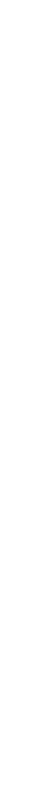


Table 1: A comparison of some different non-enzymatic glucose sensors based on different gold structures.

\begin{tabular}{|c|c|c|c|c|c|}
\hline Gold structure & $\begin{array}{c}\text { Detection potential / } \\
\mathrm{mV} \text { or technique }\end{array}$ & $\begin{array}{c}\text { Sensitivity / } \mu \mathrm{A} \mathrm{cm} \\
{ }_{2} \mathrm{mmol}^{-1} \mathrm{dm}^{3}\end{array}$ & $\begin{array}{c}\text { Linear range / } \\
\mathrm{mmol} \mathrm{dm}^{-3}\end{array}$ & $\begin{array}{c}\text { LOD / } \mu \mathrm{mol} \\
\mathrm{dm}^{-3}\end{array}$ & Reference \\
\hline Nanoparticles & LSV & 87.5 & $0.1-25$ & 50 & [8] \\
\hline Nanoparticles & & 99.11 & $2.8-6$ & - & \\
\hline Nanowire array & 200 & - & $0.4-10$ & 36 & [10] \\
\hline Dendrite-like & 150 & 190.7 & $0.1-25$ & 50 & {$[11]$} \\
\hline Nanotubes & 250 & 1.13 & 1.42 .5 & 10 & {$[14]$} \\
\hline Nanocoral & 200 & 22.6 & $0.05-30$ & 10 & [15] \\
\hline Disk & 250 & 0.72 & $0.5-20$ & 10 & [16] \\
\hline Porous film & 350 & 11.8 & $2-10$ & 5 & [17] \\
\hline Micropillar array & $\mathrm{CV}$ & 13.2 & $0.5-9$ & 60 & [18] \\
\hline Nanoparticles integrate nanotube arrays & 250 & 1.13 & $1-42.5$ & 10 & [19] \\
\hline \multirow[t]{2}{*}{ Nanowire array } & DPV & 41.9 & up to 20 & 30 & {$[24]$} \\
\hline & -400 & 309 & up to 10 & 50 & \\
\hline Flower-like & $\mathrm{CV}$ & 0.02 & $0.3-30$ & 150 & {$[25]$} \\
\hline Nanosized & SWV & 25 & up to 50 & 100 & [26] \\
\hline Hyperbranched pine-like gold nanostructure & 70 & 776.8 & $0.020-0.240$ & 3.39 & This work \\
\hline
\end{tabular}


LSV: Linear sweep voltammetry

$\mathrm{CV}$ : Cyclic voltammetry

SWV: Square wave voltammetry

DPV: Differential pulse voltammetry 
Table 2: The values of glucose levels in serum samples determined a clinical lab and obtained by the HP-Au electrode.

\begin{tabular}{cccc}
\hline $\mathrm{NO}$ & Determined in a clinical lab $/ \mathrm{mg} \mathrm{dL}^{-1}$ & Determined using HP-Au electrode $/ \mathrm{mg} \mathrm{dL}^{-1}$ & 184.2 \\
\hline 1 & 178 & 175.1 & +3.4 \\
\hline 2 & 184 & 209.4 & -4.9 \\
\hline 3 & 206 & 72.7 & +1.7 \\
\hline 4 & 76 & -4.3 \\
\hline
\end{tabular}

